\title{
The Literal Review of Panic Disorder and Comorbidity
}

\author{
Tong $\mathrm{Wu}^{1, \dagger}$ Yanni Yang ${ }^{2, *, \dagger}$ \\ ${ }^{1}$ Nanjing Foreign Language School International Center, Nanjing, China \\ ${ }^{2}$ Stony Brook University, New York, U.S.A. \\ *Corresponding author. Email: yanni.yang@stonybrook.edu
}

†These authors contribute equally.

\begin{abstract}
Considering the huge number of patients with comorbidity, this paper aims to review and summary the recent 5-years studies, from 2016 to April 2021, on the panic disorder and comorbidities. All studies that mentioned in this paper are published on authoritative databases and are peer viewed. The results of the studies that reviewed in this paper are separated into three categories: (1) the comorbidity of panic disorder and bi-polar disorder (2) the comorbidity of panic disorder and depression (3) comorbidity of panic disorder and other mental illness. The content includes the review and analysis based on psychological aspect, neuron science aspect, and medical aspect. This paper involves a few earlier studies that were published earlier than the range of recent 5 years in order to offer more evidence.
\end{abstract}

Keywords: Panic disorder, Comorbidity, Bi-polar disorder, Depression, Medicine, Treatment, Mental illness

\section{INTRODUCTION}

According to DSM-5, panic disorder is a type of anxiety disorder that is characterized by intense, recurrent, and unexpected panic attacks [1]. Research finds that about $15 \%$ of the survey respondents reported the occurrence of a panic attack over their lifetimes, and $3.8 \%$ reported a panic attack in the preceding month [2]. It is usually progressing and disabling, as well to both individuals, their families, and the whole society. However, there is a further problem with the comorbidity. For instance, there are potential negative consequences in the missing diagnosis of comorbidity. Such mistakes may lead to unexpected worse processes in the following treatment and therapy. Thus, this work aims to review and analyse the research focusing on the relationship between panic disorder and its comorbidity. Main contribution of this thesis is summarized from 2016 to April 2021. Based on these materials, comorbidity with panic disorder involved in this paper is comorbid bipolar disorder, comorbid depression, and other comorbidities (major depressive episode and generalized anxiety disorder). The content of this paper includes the reviewing of related studies and the discussing of their contributions and possible improvement that could keep on researching in the future.

\section{METHOD}

This paper focuses on a literary review and summary of the research on panic disorder's comorbidity from 2016 to April 2021. Search items were used that combined: "morbidity" and "panic disorder". The research on the comorbidity of panic disorder and bipolar disorder is from reliable sources, such as Frontiers and the MBJ. All research mentioned by this paper are published on reliable databases, such as ScienceDirect, Springer. These researches are available, peer-viewed, full-text, and published in English. All participants in all research are human beings, and the experiments used reliable tools to measure the result, such as Hamilton Depression Rating Scale (HAM-D/HDRS) and Panic Disorder Severity Scale (PDSS). After screened, 10 articles were included in this review.

\section{RESULTS}

\subsection{COMORBIDITY OF PANIC DISORDER AND BI-POLAR DISORDER}

After reviewing the literature, most studies found that panic disorder is the most common comorbidities of bipolar disorder. Bipolar disorder is a mental illness that could run in all ages, races, and genders. The onset of bipolar disorder is normally diagnosed in late 
adolescence. There are different types of bipolar disorder which are classified by the length of time, frequency of occurrence, and pattern of episodes of mania and depression. This literal review focuses on the comorbidity between bipolar I, bipolar II, and panic disorder. Considering affective temperament could influence mood disorders, there is the potential possibility that could show the relationship between panic disorder and affective temperament, such as bipolar disorder. Meanwhile, research showed that the amount of patients who have a comorbidity of panic disorder and bipolar disorder is considerable. According to Zsuzsanna Belteczki and colleagues' research, 22\% of 108 participants with panic disorder developed bipolar spectrum disorder (bipolar I or II and cyclothymic disorders), and $73 \%$ of participants who comorbid panic disorder and bipolar disorder latterly had bipolar II disorder [3]. This result implies that panic disorder has a noticeable tendency in combining with mood disorders such as bipolar disorder. Additionally, researchers further suggested that there is a higher prevalence of anxiety disorders among patients with bipolar II than bipolar I [4]. What's more, research data provided evidence of the physical similarity between panic disorder and bipolar disorder [5]. In the neuron science aspect, patients with panic disorder and bipolar disorder have shown a reduction in the prefrontal cortex activity and the activity of the amygdala. Based on previous research data, there is evidence supporting the potential association between panic disorder and bipolar disorder. Importantly, this potential relationship between panic disorder and bipolar disorder will affect the treatment outcomes of patients with panic disorder and bipolar disorder. Comorbid anxiety could lead to a worse consequence in the recovery process. For example, the comorbidity in anxiety disorder could elicit earlier relapse, especially in depression, and lead to poor quality of life [6]. More seriously, comorbidities of panic disorder and bipolar disorder are also associated with higher suicide rates [7]. The suicide prevalence for these patients is related to the degree of severe course [8]. This result means the more severe these patients' symptoms, the higher risk of suicide acts might occur. Meanwhile, the meta-analysis data indicates the high prevalent comorbidity of patients with panic disorder and bipolar disorder, implying that patients with bipolar disorder could run a lifetime course in panic disorder.

\subsection{COMORBIDITY OF PANIC DISORDER AND DEPRESSION DISORDER}

Comparing with the wide-known depression disorder in public, there are fewer studies about the comorbidity of panic disorder and depression disorder instead. However, the number of patients with panic disorder and comorbid depressive disorder is considerable. The research shows the prevalence rate of comorbid panic disorder with major depression disorder is $8.3 \%$ [9]. In the list of the costliest conditions in the United States, depression and anxiety disorder are on the top, and the cost for treating panic disorder is extreme expensive among them [10]. The patients are more likely to be young age, have more severe symptoms of panic disorder, and have increased administration of benzodiazepines (BZD) or BZD-like drugs [11]. Additionally, there is no significant difference in the effect of first-line antidepressant treatment among groups of anticipants [12]. This phenomenon could imply the possibility that antidepressants in MDD could be effective for comorbid mental illnesses [13]. Thus, patients with panic disorder and comorbidities could use antidepressants in MDD to treat all the mental illnesses spontaneously. Meanwhile, the study from Nam and colleagues proved that comorbid panic disorder was associated with a significantly increased likelihood of suicide attempts, especially at younger age. Compared to patients with single depression disorder, more patients with comorbidity panic disorders suffer from somatic comorbidity, heart disease comorbidity and asthma comorbidity [14]. Nevertheless, according to the study by Stephanie M. Gorka and the colleagues, the experiment of testing 40 anticipants (patients only with MMD, patients with MMD and comorbid panic disorder, and no lifetime diagnosis of psychopathology), participants with MDD and comorbid PD shows less activation in dorsal anterior cingulate activation (dACC) than participants only with MMD [15]. Since the activation of dACC relates to the reward anticipation deficits in MDD, the research results could imply comorbid anxiety might be a potential factor that influences the relationship between MDD and neural responses to reward anticipation [16]. As a result, though medical treatment such as BZD and BZD-like drugs can be effective on patients with panic disorder and comorbid MMD, more research about specific treatment and medicine could be future topics since there is evidence to indicate the difference occurrence in dACC among patients with or without comorbidity.

\subsection{COMORBIDITY OF PANIC DISORDER AND OTHER MENTAL ILLNESS}

According to the paper from Yapici Eser and the colleagues, the research statistically shows that the expectation for all anxiety disorder comorbidities is much higher among the public and other patients [17]. Based on Kristin Vickers and Richard J. McNally`s research, among the 8098 interviewees, all participants aged 15 to 24 with a history of more than 24 years of psychiatric disease and other interviewees were used as randomly selected samples for interviews. The result shows that among 609 interviewees who answered whether they matched at least four symptoms designed to diagnose potential panic attacks, 274 interviewees met the DSMIII-R panic disorder criteria [18]. Although the findings did not show strong evidence for the hypothesis that panic disorder itself increases the risk of suicide attempts, 
data shows that panic disorder had a potential association with the major depressive episode (MDE) and generalized anxiety disorder (GAD) [19]. Finally, considering the panic attack as one of the characteristics of agoraphobia and panic disorder, the difference between patients who suffered by panic disorder with or without comorbid agoraphobia is important for screening and diagnosis. According to Salhotra and the colleague's research, based on the result of cross-sectional observational study from anticipants who have panic disorder with or without agoraphobia, there is an obvious difference in the measurement of the severity of symptoms between these two groups [20]. The data shows the tendency to increase in the severity of panic and other anxiety symptoms in the results of comorbid psychiatric disorders and stress, which could imply the possibility of aggravating mental illness [21]. Therefore, patients who have panic disorder and comorbid agoraphobia might experience longer therapy duration to be recovery and have more severe symptoms than patients without agoraphobia.

\section{DISCUSSION}

Based on recent 5-years studies, this literal review focuses on the area of comorbidity of panic disorder and selected comorbid bi-polar disorder, anxiety disorder, depression, and generalized anxiety disorder. Firstly, the amount of patients who have a comorbidity of panic disorder and bipolar disorder is considerable. Comorbidities of panic disorder and bipolar disorder are also associated with higher suicide rates and there is a potential negative consequence in the recovery process. Besides, there is a physical similarity between panic disorder and bipolar disorder in the reduction of certain brain activities (the prefrontal cortex activity and the activity of the amygdala). Secondly, the number of patients with panic disorder and depression is quite large since the prevalence is $8.3 \%$ based on Dold and the colleagues' study. Also, the mean of these patients is lower than that for other patients with different mental illnesses, which indicates a younger age tendency in prevalence. In the former study, BZD and BZD-like drags show potential effectivity on both patients with and without comorbidity of panic disorder and major depressive disorder. However, since the test about dACC shows the influence of comorbid anxiety among patients with major depressive disorder, and study shows the severity of suicide attempt and suffering of other illness such as somatic comorbidity, heart disease comorbidity and asthma comorbidity among patients with panic disorder and comorbid depression, there is a new field that focuses on researching specific medicine and treatment for patients with panic disorder and major depressive disorder. Finally, for other comorbidities, all anxiety disorder comorbidities are much higher among the public and other patients. In the neuron science aspect, panic disorder had a potential association with MDE and
GAD. In psychological aspect, patients who have comorbid agoraphobia experience more severe symptoms that patients without this comorbidity. However, this literal review exists some limitations. The study result from Vickers, K., \& McNally, R. J.'s research was published in 2004, which is 17 years ago comparing with the data of this literal review. Thus, this study result might be antiquated. Nevertheless, all the studies, including Vickers, K., \& McNally, R. J.'s research, are published in an authoritative database and peer-reviewed. As a result, all materials in this literal review are reliable. Based on the content of these studies, there is a potential application in offering a summary of recent 5-year studies about the comorbidity of panic disorder and identifying the topic of deeper studies on the comorbidity of panic disorders, such as updated research about whether comorbid MED and GAD is the risk factor in increasing suicide attempts.

\section{CONCLUSION}

The purpose of this paper is to review and to analyse recent studies on the comorbidity of panic disorder. It aims to understand latest knowledge of this field and find potential area that researchers could explore deeper to help these patients to get a better healing process and life status. Based on these studies, this paper involves the facts of high prevalence, and large number of patients with panic disorder and comorbidities are suffering. It reviews comorbid bi-polar disorder, depression, and other mental illnesses. There is an association between panic disorder and comorbidities, and they play roles in influencing the process of therapying and healing for patients. This paper also reviews and discusses the efficacy of medicines. For example, the potential application could be the enlighten in making different therapy plans and offering medicine for patients who have panic disorder with or without agoraphobia. The conclusion is that there could be more specific medicines for these patients in the future.

\section{REFERENCES}

[1] American Psychiatric Association. (2013). Diagnostic and statistical manual of mental disorders (DSM-5®). American Psychiatric Pub.

[2] Eaton, W. W., Kessler, R. C., Wittchen, H. U., \& Magee, W. J. (1994). Panic and panic disorder in the United States. The American journal of psychiatry

[3-5] Belteczki, Z., Rihmer, Z., Rozsa, S., Ujvari, J., Pompili, M., Gonda, X., \& Dome, P. (2021). Affective Temperaments, Panic Disorder and Their Bipolar Connections. Medicina (Kaunas, Lithuania), 57(3), 289-. 
[6] Coryell W, Solomon DA, Fiedorowicz JG, et al. Anxiety and outcome in bipolar disorder. Am J Psychiatry 2009;166:1238-43

[7-9] El-Mallakh, R., \& Hollifield, M. (2008). Comorbid Anxiety in Bipolar Disorder Alters Treatment and Prognosis. Psychiatric Quarterly, 79(2), 139-150. https://doi.org/10.1007/s11126-008-9071-5

[10] Roehrig C. 2016. Mental disorders top the list of the most costly conditions in the United States: \$201 billion. Health Aff. 35(6):1130-35

[11-13] Dold, M., Bartova, L., Souery, D., Mendlewicz, J., Serretti, A., Porcelli, S., ... \& Kasper, S. (2017). Clinical characteristics and treatment outcomes of patients with major depressive disorder and comorbid anxiety disorders-results from a European multicenter study. Journal of psychiatric research, 91, 1-13.

[14] Nam, Y. Y., Kim, C. H., \& Roh, D. (2016). Comorbid panic disorder as an independent risk factor for suicide attempts in depressed outpatients. Comprehensive psychiatry, 67, 13-18.

[15-16] Gorka, S., Huggins, A., Fitzgerald, D., Nelson, B., Phan, K. and Shankman, S., 2014. Neural response to reward anticipation in those with depression with and without panic disorder. Journal of Affective Disorders, 164, pp.50-56

[17] Yapici Eser, H., Kacar, A. S., Kilciksiz, C. M., Yalçinay-Inan, M., \& Ongur, D. (2018). Prevalence and associated features of anxiety disorder comorbidity in bipolar disorder: a meta-analysis and meta-regression study. Frontiers in psychiatry, 9, 229.

[18-19] Vickers, K., \& McNally, R. J. (2004). Panic Disorder and Suicide Attempt in the National Comorbidity Survey. Journal of Abnormal Psychology, 113(4), 582-591. https://doiorg.proxy.library.stonybrook.edu/10.1037/0021843X.113.4.582

[20-21] Salhotra, N., Bhattacharyya, D., Wadhawan, J., \& Yadav, P. (2018). Sociodemographic and Clinical Variables related to Panic Disorder with and without Agoraphobia. Journal of Clinical and Diagnostic Research, 12(4), VC01-VC04. https://doi.org/10.7860/JCDR/2018/30266.11429 